\title{
A Novel Vehicle Formulation for Treatment of Inflammatory Skin Diseases
}

\author{
A. Zemtsov ${ }^{1}$, H. Hosier ${ }^{2}$ \\ ${ }^{1}$ Department of Dermatology, School of Medicine, Indiana University, Bloomington, USA; ${ }^{2}$ Dermatology Center Clinical Trials Section, \\ Indiana University, Bloomington, USA. \\ Email: azemtsov@iupui.edu
}

Received November $14^{\text {th }}, 2012$; revised December $17^{\text {th }}, 2012$; accepted December $26^{\text {th }}, 2012$

\begin{abstract}
Objective: To test the efficacy of a new topical vehicle formulation containing cetirizine in emu oil in treating inflammatory skin conditions. Methods: A single blind half body comparative study of patients with psoriasis, atopic and stasis dermatitis were all treated with midpotency topical steroid (desoximethasone) in propylene glycol base, desoximethasone and cetirizine in propylene glycol base, desoximethasone in emu oil base and desoximethasone with cetirizine dissolved in emu oil. Results: Based on patients' ranking of creams' efficacy, and supported by photographic data and investigators' clinical assessment, results clearly indicated that desoximethasone and cetirizine in emu base was statistically more efficacious than either desoximethasone alone or with cetirizine in neutral propylene glycol base or desoximethasone in emu oil base (without cetirizine). Conclusions: Cetirizine and emu oil, having inherent anti-inflammatory and other beneficial properties synergistically, and by different biochemical pathways, enhance and magnify each other's pharmaceutical effects that are useful in treatment of skin inflammatory diseases.
\end{abstract}

Keywords: Cetirizine; Antihistamines; Emu Oil; Psoriasis; Eczema; Atopic Dermatitis

\section{Introduction}

Extensive investment of time and capital is required to obtain FDA approval to sell and market a new drug; this is a reason that, in the past 5 years, only three topical dermatological drugs were approved by the FDA $[1,2]$. Instead, pharmaceutical companies are primarily directing their efforts to develop and patent new delivery systems and formulations to more efficiently carry active ingredients (drugs) through the stratum corneum skin barrier. These new formulations include solid lipid nanoparticles, liposomes and niosomes, transferosomes, ethosomes, cyclodextrins, and sol-gel microcapsules [1,2].

The novel approach illustrated in this study is to develop a formulation that in itself is a therapeutic agent in addition to its skin penetrating and emulsifying properties. As is further addressed in the discussion section, cetirizine dissolved in emu oil can either be a vehicle to dissolve and to carry an active ingredient through skin barrier and then synergistically to potentiate its therapeutic effects, or a pharmaceutical product by itself.

This formulation, developed for this study, contains 4 ingredients: emu oil $>85 \%$ by weight, cetirizine nonsedating antihistamine $2 \%$ by weight, methylparaben preservative $0.2 \%$, and propylene glycol that potentiates emu oil solvent and emulsifying properties.
Emu oil has excellent skin barrier penetrating features [3]. These skin-penetrating properties are due to oleic acid (over $50 \%$ of emu oil content by weight is an oleic acid). Oleic acid is known as a skin penetration enhancer and works by fluidizing the intercellular lipids of the stratum corneum [3]. Two other valuable properties of emu oil as a formulation skin delivery system are its superb emulsifying properties and low potential for irritation [4]. Most importantly, emu oil, in addition to formulation properties mentioned above, has anti-inflammatory features; it has been shown that topically administered emu oil is as effective as orally administered high dose ibuprofen [5]. Furthermore, it is believed that at least some of emu oil's anti-inflammatory properties are due to tumor necrosis factor-alpha inhibition [6]. Other therapeutically useful properties of emu oil include excellent moisturizing qualities [7], antibacterial and noncomedogenic features [4].

Cetirizine, being an antihistamine, obviously has antipruritic properties that are very beneficial in treating skin inflammatory conditions. Furthermore, because it is a second-generation nonsedating antihistamine, it has an excellent safety profile. However, the most important and unique feature of cetirizine is that it has unique and numerous anti-inflammatory properties such as inhibition adhesion molecules both in atopic dermatitis [8] and pso- 
riasis [9], inhibition of both $\mathrm{T}$ lymphocytes and monocytes [10], and many other anti-inflammatory features. Moreover, these anti-inflammatory properties of cetirizine are totally separate from its $\mathrm{H} 1$ antihistamine features [11]. Thus, the principal investigator of this study (AZ) theorized that emu oil and ceterizine will act synergistically and potentiate each other's beneficial effects in treating inflammatory skin conditions by utilizing completely different biochemical pathways, and this study was designed to prove this hypothesis. Furthermore, emu oil, in addition to being a therapeutic agent, also acted as a skin delivery system for cetirizine and topical steroid.

\section{Materials and Methods}

Study procedure: this study was designed as a single blind study. Namely, all research subjects received 4 tubes of cream labeled as cream A, B, C or D. However, both principal investigator and study coordinator knew that cream A was desoximethasone and ceterizine in emu oil base (all 4 creams also had, as stated above, methylparaben as a preservative and various amounts of propylene glycol as a neutral emulsifying solvent). Cream B was desoximethasone in propylene glycol, cream $\mathrm{C}$ was desoximethasone in emu oil, and cream D was desoximethasone and cetirizine in propylene glycol base. Obviously, all 4 creams had an identical amount of desoximethasone $(0.25 \%$ by weight). To improve statistical significance of the results, this was a comparative half/ split body study; namely, each research subject was applying on the diseased skin on one side of the body cream A and on the identical abnormal skin on the opposite side of the body either cream B, C or D. Thus, each research subject was its own positive and negative control. At the end of the study, each research subject was asked to complete a questionnaire specifically asking "which of 4 creams worked the best" and numerically ranking efficacy of each cream on a scale of 1 (not effective) to 10 (extremely effective). Furthermore, they were asked to report any systemic or dermatological side effects of medications such as irritation, stinging or pruritus. Moreover, the subject's clinical response was assessed by a principal investigator at the beginning and the end of the clinical trial. Finally, the study coordinator obtained clinical photographs of all areas treated at the beginning and end of this study.

Subjects: ten [10] patients enrolled and all completed the study. The patients did not use any other prescription or over the counter topical preparation during the duration of this study. Informed written consent was obtained prior to initiation of the study from each research subject. No financial compensation was provided to any of the research subjects. However, each study participant received a free complimentary office visit and free medication. Furthermore, at the end of the trial, patients were offered and received a free complimentary tube of the cream of their choice (either cream D, B, A or C). The patients' clinical diagnoses were either psoriasis, atopic or stasis dermatitis.

\section{Results}

All 10 subjects ranked cream A as the most effective for treatment of their disease $(p<0.05$; statistically significant result). Furthermore, all 10 study participants, at the end of the study, requested an additional free tube of cream A only $(<0.05)$. In evaluating patients' numerical scores of creams' efficacy, cream A, C and D was compared to cream B (steroid cream in neutral propylene glycol base). Again cream A had the highest score in comparison to creams C, D and B ( $<<0.05)$. Finally, clinical evaluation by a principal investigator collaborated the results of patients' questionnaire. For example, subject 2, with chronic atopic dermatitis on the neck unresponsive to ultrapotent clobetasol steroid cream, had complete clearance of her rash after 5 day therapy with cream A. Subject 5, with chronic severe plaque psoriasis under reasonable control with subcutaneous etanercept injections, had persistent psoriatic lesions on her abdomen unresponsive to therapy with potent fluocinonide steroid cream; her disease completely cleared within 15 days therapy with cream A.

None of the research subjects reported any systemic side effects to any of the 4 creams. As far as cutaneous dermatological side effects are concerned, one patient reported mild pruritus associated with cream A use; nonetheless, she still gave cream A the highest ranking in all categories and requested an additional cream A tube. None of other 9 participants reported dermatological side effects associated with cream A use. These results unequivocally demonstrate that emu oil and cetirizine synergistically potentiate each other's beneficial effects and these results are statistically significant.

\section{Discussion}

Atopic dermatitis affects between 10 and 20 percent of the US population, causing considerable morbidity, poor quality of life, and high medical costs to both patients and society [12]. Topically administered steroid creams are the main form of therapy, but prolonged use of these glucocorticoid agents is associated with skin thinning, permanent stretch marks, atrophy, rebound effects, tachyphylaxis, and potential systemic absorption that can cause numerous side effects [13]. Topical calcineurin inhibitors (pimecrolimus and tacrolimus) are effective alternatives but are infrequently used since the FDA issued a black box warning concerning a potential increase in systemic malignancies in patients using these topical preparations [13]. Psoriasis is also a chronic inflammatory disease 
affecting $2 \%$ of the US population; based on the severity of the disease, topical steroids, vitamin D analogs, either by themselves or in combination with phototherapy, or systemic agents such as methotrexate, cyclosporin A or biological agents are used for therapy [14]. Clearly a new anti-inflammatory, antipruritic, moisturizing formulation/ topical agent would be both a significant and welcome addition to the therapeutic armaments used in treating atopic dermatitis, psoriasis and other inflammatory skin conditions.

This pilot single-blinded, half body/mirror image study unambiguously demonstrated that cetirizine in emu oil base, in addition to being an excellent skin delivery formulation is in its own right, is an effective therapeutic agent for treatment of skin inflammatory diseases. Therefore, it would make sense to use cetirizine/emu oil in combination with glucorticoids for a short period of time and for localized areas to clear active inflammatory lesions, and then use cetirizine/emu oil combination as a moisturizer to prevent recurrence of the disease and thus avoid the side effects of chronic topical steroids mentioned above.

As stated in Introduction, it is assumed that anti-inflammatory properties of emu oil are at least in part due to inhibition of tumor necrosis factor-alpha inhibition, a key target inflammatory molecule in systemic psoriasis therapy; cetirizine is anti-inflammatory at least in part due to inhibition of adhesion molecules [6,8,9]. Emu oil has excellent moisturizing properties. Moisturizers by themselves are therapeutic agents in treatment of patients with psoriasis, atopic dermatitis and other forms of eczema; they improve skin hydration and normalize keratinocyte differentiation [15]. Moreover, bacterial secondary colonization and infection play important roles in pathogenesis of atopic dermatitis; emu oil antibacterial properties addresses this problem [4]. Finally, antipruritic properties of cetirizine are obviously beneficial in treatment of most skin inflammatory diseases. This study clearly demonstrated that cetirizine and emu oil synergistically potentiate each other with mutually beneficial effects in treating skin inflammatory conditions and by utilizing completely different biochemical pathways. As stated in Introduction section, emu oil is unique among organic oils by being totally noncomedogenic; the authors are in the process of conducting a study combining cetirizine/emu oil formulation with topical tretinoin for treatment of acne and preliminary results, as with psoriasis and eczema patients in this study, are very encouraging. Finally, we are studying whether cetirizine/emu oil formulation in combination with creatine and creatinine would be beneficial in wound healing.

\section{REFERENCES}

[1] S. Fireman, O. Toledano, K. Niemann, N. Loboda and N.
Dayan, "A Look at Emerging Delivery Systems for Topical Drug Products," Dermatologic Therapy, Vol. 24, No. 5, 2011, pp. 477-488. doi:10.1111/j.1529-8019.2012.01464.x

[2] W. H. Eaglstein and G. Corcoran, "New Drugs and New Molecular Entities in Dermatology," Archives of Dermatology, Vol. 147, No. 5, 2011, pp. 568-572.

doi:10.1001/archdermatol.2011.100

[3] L. H. Kircik, J. B. Bikowski, D. E. Cohen, Z. D. Draelos and A. Herbert, "Vehicles Matter; Formulation Development, Testing and Approval," Practical Dermatology, Vol. 1, No. 1, 2010, pp. 3-16.

[4] S. Farmer, "Emu Oil and Its Various Fractions as a Carrier for Antifungal, Antibacterial, and Antiviral Medications and Preparations," US Patent 7048950, 2006.

[5] J. M. Snowden and M. W. Whitehouse, "Antiinflammatory Activity of Emu Oil in Rats," Inflammopharmacology, Vol. 5, No. 2, 1997, pp. 127-132. doi:10.1007/s10787-997-0021-X

[6] X. W. Qui, J. H. Wang, X. W. Fang, Z. Y. Gong, Z. Q. Li and Z. H. Yi, "Anti-Inflammatory Activity and Healing-Promoting Effects of Topical Application of Emu Oil," Academic Journal of the First Medical College of PLA, Vol. 25, No. 4, 2005, pp. 407-411.

[7] A. Zemtsov, M. Gaddis and V. M. Montalvo-Lugo, "Moisturizing and Cosmetic Properties of Emu Oil: A Pilot Double Blind Study," Australasian Journal of Dermatology, Vol. 37, No. 3, 1996, pp. 159-161. doi:10.1111/j.1440-0960.1996.tb01040.x

[8] M. Boone, L. Lespagnard, N. Renard, M. Song and J. P. Rihoux, "Adhesion Molecule Profiles in Atopic Dermatitis vs. Allergic Contact Dermatitis: Pharmacologic Modulation by Cetirizine," Journal of European Academy of Dermatology and Venereology, Vol. 14, No. 4, 2000, pp. 263-266. doi:10.1046/j.1468-3083.2000.00017.x

[9] E. Pestelli, I. Florianati, P. Fabbri and M. Caproni, "Cetirizine Modulates Adhesion Molecule Expression in a Double-Blind Controlled Study in Psoriatic Patients," International Journal of Tissue Reactions, Vol. 25, No. 1, 2003, pp. 1-8.

[10] T. Jinquan, C. M. Reimert, B. Deleuran, C. Zachariae, C. Simonsen and K. Thestrup-Pederssen, "Ceterizine Inhibits the in Vitro and ex Vivo Chemotactic Response of T Lymphocytes and Monocytes," Journal of Allergy and Clinical Immunology, Vol. 95, No. 5, 1995, pp. 979-986. doi:10.1016/S0091-6749(95)70098-6

[11] M. Koller, R. A. Hilger, J. P. Rihoux and W. Konig, "Cetirizine Exerts Anti-Inflammatory Effects on Human Neutrophils," International Archives of Allergy and Immunology, Vol. 110, No. 1, pp. 52-56.

[12] A. Shobailli, "The Impact of Childhood Atopic Dermatitis on the Patient Family," Pediatric Dermatology, Vol. 27, No. 6, pp. 618-623. doi:10.1111/j.1525-1470.2010.01215.x

[13] B. A, Yentzer, R. A. Ade, J. M. Fountain, A. R. Clark, S. R. Taylor, E. Bonderding and S. R. Feldman, "Improvement in Treatment Adherence with a Three-Day Course of Fluocinonide Cream $0.1 \%$ for Atopic Dermatitis," $\mathrm{Cu}$ tis, Vol. 86, No. 4, pp. 208-213. 
[14] American Academy of Dermatology, "Psoriasis and Psoriatic Arthritis," 2010.

[15] J. W. Fluhr, C. Cavalotti and E. Benardesca, "Emollient
Moisturizers and Keratolytic Agents in Psoriasis," Clinics in Dermatology, Vol. 26, No. 4, 2008, pp. 380-386. doi:10.1016/j.clindermatol.2008.01.015 